\title{
On the Theory of the Virial Development of the Equation of State of Monoatomic Gases
}

\author{
R. J. RIDDELl, JR., ${ }^{*}$ Department of Physics and Radiation Laboratory, University of California, Berkeley, California \\ AND \\ G. E. Uhlenbeck, H. M. Randall Laboratory of Physics, University of Michigan, Ann Arbor, Michigan
}

(Received May 26, 1953)

\begin{abstract}
The problem of the condensation of a gas is intimately related to the asymptotic behavior of the vurial coefficients, $B_{m}$, as $m \rightarrow \infty$. The problem of the evaluation of the virial coefficients may be divided into two distinctly different ones. The first of these, which is purely combinatorial in nature and is independent of the intermolecular force law, is that of determining the number of a certain type of connected graphs of $l$ points and $k$ lines which are called "stars." This problem is solved by means of generating functions, wi th the result that the total number of such stars is asymptotically equal to
\end{abstract}

$$
\left(\begin{array}{c}
\frac{1}{2} l(l-1) \\
k
\end{array}\right)
$$

for almost all $k$. Arguments are also presented which indicate that the total number of topologically different stars is

$$
\frac{1}{l !}\left(\begin{array}{c}
\frac{1}{2} l(l-1) \\
k
\end{array}\right)
$$

With these results the combinatorial problem is essentially solved.

The second problem is that of evaluating certain integrals of functions which depend on the intermolecular potental. This problem is not so near to a solution. For a purely repulsive force, asymptotic expressions are obtained for $k=l$, and $k=l+1$. The partial contributions to the virial coefficient in these two cases are.

and

$$
(-1)^{l} \cdot \frac{5}{3}\left(\begin{array}{c}
5 \\
2 \pi
\end{array}\right)^{l}(2 b)^{l-1} \frac{(l-1)}{l^{5 / 2}}
$$

$$
(-1)^{l} \frac{2 \cdot 5^{3}}{24 \pi \sqrt{3}}(2 b)^{l-1}
$$

respectively. Results for some simple one-dimensional rigid lines are also given.

\section{INTRODUCTION}

$\mathbf{M}^{\circ}$ OST of the explicit theories of the condensation of gases are based on the development of the thermodynamic quantities of the gas in powers of the density or of the inverse of the specific volume. ${ }^{1}$ This leads to the well-known virial development of Kamerlingh Onnes for the equation of state:

$$
p v=R T\left[1+\frac{B_{2}(T)}{v}+\frac{B_{3}(T)}{v^{2}}+\cdots\right]
$$

in which the deviations of the ideal gas law due to the interaction of the molecules in pairs, triples, etc., are taken successively into account in the successive virial coefficients $B_{2}(T), B_{3}(T)$, etc. The theory gives explicit expressions for these virial coefficients in terms of the intermolecular forces, but the actual evaluation of the integrals is notoriously involved and seems quite out of the question for the higher coefficients. The condensation phenomenon depends on the convergence of the series

* U. S. Atomic Energy Commission Predoctoral fellow, University of Michigan, 1950-1951.

${ }^{1}$ We have especially in mind the theory of J. E. Mayer; for a summary see his book: Statistical Mechanics (John Wiley and Sons, Inc., New York, 1940), Chap. 13 Compare also B. Kahn and G. E. Uhlenbeck, Physica 5, 399 (1938) ; B. Kahn, dissertation, University of Utrecht, 1938; J. de Boer, dissertation, University of Amsterdam, 1940; K. Husimi, J. Chem. Phys. 18, 686 (1950).
(1) ${ }^{2}$ however, and therefore on the asymptotic behavior of the $B_{l}(T)$ for high $l$, and we thought that this asymptotic behavior might be simpler to determine, especially for simple force laws, as, for instance, for a gas of elastic spheres.

The motivation to consider especially the case of elastic spheres was the striking and paradoxical result of Kirkwood and co-workers ${ }^{3}$ who showed that on the basis of the so-called superposition approximation, a gas of elastic spheres has a transition point at a volume of about 1.24 times the volume of close packing. Although perhaps hard to believe, one must admit that there is no rigorous argument which disproves the existence of such a condensation point, and, since the work of Onsager, one has learned to be rather skeptical about intuitive and approximate arguments. In addition there is the suggestion that perhaps the Kirkwood transition has something to do with the solidification of helium, which is known to occur at temperatures many times the critical temperature, so that it is hard to ascribe it to the weak attractive forces between the helium atoms which are of the order $k T_{\text {crit }}$. The solidification of a gas is

${ }^{2}$ Or better on the convergence of the series $\Sigma b_{l} z^{l}$, where $b_{l}$ are the Mayer cluster integrals from which the virial coefficients follow. See Sec. II.

${ }^{3}$ J. G. Kirkwood and E. Monroe, J. Chem. Phys. 9, 514 (1941). Kirkwood, Maun, and Alder, J. Chem. Phys. 18, 1040 (1950) See also M. Born and H. S. Green, A General Kinetic Theory of Lequids (Cambridge University Press, Cambridge, 1949) 
perhaps a general consequence of the sharp repulsive forces, which in first approximation may well be idealized by the elastic sphere model.

On the other hand, the argument of Kirkwood is surely not rigorous either, since it depends on his superposition approximation, ${ }^{4}$ and the only way we know of to arrive at a rigorous result is through the convergence discussion mentioned above. Unfortunately, we have not been able to solve the problem of the asymptotic behavior of the $b_{l}$ and $B_{l}$, so that the question of the existence of a transition point for a gas of elastic spheres remains open. We would like to report on some partial results. In Sec. II we will recapitulate the general formula for the cluster integrals $b_{l}$ and for the virial coefficients $B_{l}$, always with the assumption that the intermolecular forces are additive. In this case the expressions for $b_{l}$ and $B_{l}$ consist, for large $l$, of a great number of different integrals over the configuration space of $l$ particles. Therefore two problems can be distinguished; the first one is to determine the number of different integrals and their multiplicities, and the second is to calculate the value of each "irreducible" integral. Such a distinction is of course common to any successive approximation method, and from the Feynman diagrams used in the perturbation theory of quantum mechanics it has become a familiar fact that the first problem can always be formulated as a problem in the theory of linear graphs. One has to count all possible topologically different graphs of a certain kind. In Sec. III the precise formulation of the combinatorial problems will be given in the language of the theory of graphs, and in Secs. IV and V the solution of these problems will be discussed, with special emphasis on the asymptotic behavior for large $l$ of the different numbers involved. ${ }^{5}$ The results are of course independent of the form of the intermolecular potential. This enters in the second and more difficult problem, the calculation of the irreducible integrals. We have made no progress with this problem, and in Sec. VI only some remarks and suggestions will be presented.

\section{GENERAL EXPRESSIONS FOR THE CLUSTER INTEGRALS AND VIRIAL COEFFICIENTS}

For additive intermolecular forces one can write the partition function for $N$ particles in the volume $V$, following Mayer, in the form:

$$
\begin{aligned}
& Z=\frac{1}{N !}\left(\frac{2 \pi m k T}{h^{2}}\right)^{3 N / 2} \\
& \quad \times \int_{V} \cdots \int_{V} d^{3} r_{1} \cdots d^{3} r_{N} \prod_{i<j=1}^{N}\left(1+f_{i j}\right)
\end{aligned}
$$

4 For a criticism of this approximation with regard to the radial distribution function and the value of the fourth virial coefficient for a gas of elastic spheres see B. R. A. Nijboer and L. van Hove, Phys. Rev. 85, 777 (1952).

5 Thanks to a communication of Professor G. Polya the actual counting problems for finite $l$ are almost completely solved also. We will mention the results but omit the proofs, since the asymptotic behavior can be seen by more intuitive arguments. For a where $f_{v_{j}}=\exp \left(-\phi\left(r_{i j}\right) / k T\right)-1$, and the product goes over all possible pairs. Consider first the product:

$$
\prod_{\imath<j=1}^{N}\left(1+f_{\imath}\right)
$$

for $l$ particles. If one expands the product, one clearly gets $2^{\frac{i}{i} l(l-1)}$ terms, and each term can be represented by a linear graph if one indicates each particle by a point and each factor $f_{i j}$ by a line between particles $i$ and $j$. The different terms can first be grouped according to the number of factors $f_{i j}$, that is, according to the number, $k$, of lines in the graph. One can further distinguish between

(a) Separated graphs, consisting of two or more graphs which are not connected together, and

(b) Connected graphs. The so-called cluster function $U_{l}\left(\mathbf{r}_{1}, \mathbf{r}_{2}, \cdots, \mathbf{r}_{l}\right)$ is the sum of all terms represented by connected graphs of $l$ points and the cluster integral is then defined by:

$$
b_{l}=\frac{1}{l ! V} \int_{\psi} \cdots \int_{V} d^{3} r_{1} \cdots d^{3} r_{l} U_{l}\left(\mathbf{r}_{\mathbf{1}}, \mathbf{r}_{2}, \cdots, \mathbf{r}_{l}\right)
$$

For fixed $l$ and large volume the $b_{l}$ become asymptotically independent of $V$, and one can show then that $Z$ becomes a polynomial of degree $N$ in $V$, given by :

$$
Z=\left(\frac{2 \pi m k T}{h^{2}}\right)^{3 N / 2} \underset{(m \ell)}{S_{l}^{\prime}} \prod_{l} \frac{\left(V b_{l}\right)^{m l}}{m_{l} !}
$$

where the round summation sign indicates a sum over all sets of integers $m_{1}, m_{2}, \cdots$ with the condition (indicated by the prime):

$$
\sum_{l=1}^{N} l m_{l}=N
$$

Since for large $N$ the partition function $Z$ is related to the free energy per particle $\psi(v, T)$, with $v=\lim (V / N)$ by : 6

$$
Z=\exp (-N \psi / k T)
$$

one can then derive the well-known Mayer equations:

$$
\begin{aligned}
& p=k T \sum_{l} b_{l} z^{l}, \\
& 1 \\
& -=\sum_{l} l b_{l} z^{l},
\end{aligned}
$$

which express the pressure and the specific volume as power series in the variable $z$, which is related to the chemical potential of the gas.

To obtain the virial development for the equation of state one must elimlnate $z$ between the two Eqs. (6).

similar investigation of the number of different Feynman diagrams in various field theories, see C. A Hurst, Proc. Roy. Soc. (London) 214, 44 (1952); also, R. J. Riddell, Jr., Phys. Rev, 91, 1243 (1953). - For a rigorous proof of these statements, see L. van Hove, Physica 15, 951 (1949). 
Formally this can be done as follows: define a set of quantities $\beta_{\nu}(T), \nu=1,2, \cdots$ by the prescription that $l^{2} b_{l}$ is to be the coefficient of $t^{l-1}$ in $\exp \left(l \sum \beta_{v} t^{\nu}\right)$. This gives, for instance:

$$
\begin{aligned}
& b_{2}=\frac{1}{2} \beta_{1}, \\
& b_{3}=\frac{1}{3} \beta_{2}+\frac{1}{2} \beta_{1}^{2}, \\
& b_{4}=\frac{1}{4} \beta_{3}+\beta_{1} \beta_{2}+\frac{2}{3} \beta_{1}^{3},
\end{aligned}
$$

and so on. One can solve successively for the $\beta_{\nu}$ in terms of the $b_{l}$, and thus one sees that the two sets of quantities $b_{l}$ and $\beta_{v}$ are uniquely related to each other. Then one can show ${ }^{7}$ that from (6) follows:

$$
\frac{p}{k T}=\frac{1}{v}-\sum_{\nu=1}^{\infty} \frac{\nu}{\nu+1} \beta_{\nu} \frac{1}{v^{\nu+1}},
$$

so that the $l^{\prime}$ th virial coefficient $B_{l}$ is given by

$$
B_{l}=-\frac{(l-1)}{l} \beta_{l-1}
$$

The question arises how to express the $\beta_{\nu}$ as an integral over the configuration space of $\nu$ particles, analogous to the expression (3) for $b_{l}$. To do this one has to separate the terms represented by connected graphs, which enter in $U_{l}$, in two groups in the following way. An articulation point of a graph is a point where the graph could be cut ${ }^{8}$ into two or more separated graphs by cutting all the lines going to this point. A connected graph which has no articulation points will be called a star. From the terms represented by connected graphs of $l$ points, select the terms which are represented by stars. The sum of these terms will be called the star function, $V\left(\mathbf{r}_{1}, \mathbf{r}_{2}, \cdots, \mathbf{r}_{l}\right)$, and Mayer proves that:

$$
\beta_{\nu}=\frac{1}{\nu ! V} \int_{V} \cdots \int_{V} d^{3} \mathbf{r}_{1} \cdots d^{3} r_{\nu+1} V\left(\mathbf{r}_{1}, \cdots, \mathbf{r}_{\nu+1}\right) .
$$

Equations (3) and (9) are the starting point of our considerations.

\section{COMBINATORIAL PROBLEMS IN THE THEORY OF GRAPHS ${ }^{9}$}

The simplest type of connected graph is the so-called Cayley tree. They consist of $l$ points and $l-1$ lines, from which follows that there are no closed loops in a Cayley tree. A more general type of connected graph may be called Husimi trees, since they were first introduced in a paper by Husimi. ${ }^{10}$ A Husimi tree is a connected graph in which no line lies on more than one cycle. It is

\footnotetext{
${ }^{7}$ For the proof see B. Kahn and G. E. Uhlenbeck, reference 1. ${ }^{8}$ Therefore it is also often called a "cutting point."

${ }^{9}$ For the general topological theory of linear graphs see the book of Koenig, Theorie der Endlichen und Unendlichen Graphen (Leipzig, 1936). Combinatorial questions are not discussed in this book. For these the basic reference is the paper of G. Polya, Acta Math. 68, 145 (1938)

${ }^{10}$ K. Husimi, J. Chem. Phys. 18, 682 (1950).
}

characterized by the numbers $n_{2}, n_{3}, n_{4}, \cdots$ of lines, triangles, quadrilaterals, etc., out of which it is built up. The number of points, $l$, is equal to $1+n_{2}+2 n_{3}+\cdots$. A pure Husimi tree is one which consists of only one type of figure. Clearly a Cayley tree is a special case of a pure Husimi tree.

A general connected graph is divided by its articulation points into a number of stars. If in each star one would disregard all internal lines and consider only the "outline" one would obtain a Husimi tree. In this sense the general connected graph is a generalization of the Husimi tree, just as a star is a generalization of a polygon.

There are two kinds of combinatorial problems connected with each type of graph. The first and simplest kind of problem is to find the number of different graphs if all points are distinguished from each other. For Cayley trees of $l$ points, the answer ${ }^{11}$ is simply $l^{l-2}$. For Husimi trees of $l$ points and type $n_{2}, n_{3}, \cdots$ the answer ${ }^{10}$ is:

$$
\frac{l !}{\prod_{l=2}^{l}[(i-1) !]^{n_{\imath}} n_{\imath} !} l^{\sum_{i=2}^{l} n_{2}-2}
$$

which clearly specializes to $l^{l-2}$ for Cayley trees. In the next section we will determine the numbers $d(l, k)$ and $c(l, k)$ of separated (or disjoint) and of connected graphs of $l$ points and $k$ lines, always assuming that all points are distinguished from each other. Clearly:

$$
d(l, k)+c(l, k)=\left(\begin{array}{c}
\frac{1}{2} l(l-1) \\
k
\end{array}\right) .
$$

In addition we will find the fraction of the connected graphs which are stars. Let $s(l, k)$ denote their number.

The numbers $c(l, k)$ and $s(l, k)$ for all possible values of $k$ indicate how many terms are involved in the cluster and star function of $l$ points. However if one integrates over the configuration space of the $l$ points, all terms which differ only in the labelling of the $l$ points will clearly give the same results. To find the number of "irreducible" integrals in (3) and (9) one has to determine the number of connected graphs and stars which are really different. This brings us to the second and more difficult kind of problem: to find the number of topologically different graphs of a certain type if the points are not distinguishable. For Cayley trees the problem has been solved in all details, ${ }^{12}$ and a beginning has been made for the case of Husimi trees of type $n_{2}, n_{3}, \cdots .^{13}$ In Sec. $\mathrm{V}$ we will give the answer for the numbers $\delta(l, k)$ and $\gamma(l, k)$ of separated and of con-

${ }^{11}$ A. Cayley, Collected Mathematical Papers (Cambridge 18891898) Vol. 13, p 26; other proofs are given by Polya, reference 9 , and by G. Bol, Abhardl. Math. Seminar Hamburg 12, 242 (1938). ${ }^{12} \mathrm{R}$ Otter, Ann. Math 49, 583 (1948).

${ }^{13}$ F. Harary and G. E. Uhlenbeck, Proc. Natl. Acad. U. S. 39, 315 (1953). 
nected graphs of $l$ points and $k$ lines which are topologically different. The corresponding number, $\sigma(l, k)$, for stars is not yet known.

We will denote by $d_{\imath}(l, k), c_{\imath}(l, k)$, and $s_{\imath}(l, k)$ the number of separated graphs, connected graphs, and stars of $l$ points and $k$ lines and of a definite topological type indicated by the index $i$, so that:

$$
\begin{aligned}
& d(l, k)=\sum_{i=1}^{\delta_{(l, k)}} d_{\imath}(l, k), \\
& c(l, k)=\sum_{i=1}^{\gamma(l, k)} c_{\imath}(l, k), \\
& s(l, k)=\sum_{i=1}^{\sigma(l, k)} s_{\imath}(l, k) .
\end{aligned}
$$

As an example we have drawn in Fig. 1 all topologically different graphs of five points, arranged according to the value of $k$. The numbers in parentheses refer to the number of times the graph occurs, and represent therefore the $d_{2}(5, k), c_{2}(5, k), s_{2}(5, k)$. One can also read off the numbers $\delta(5, k), \gamma(5, k)$, and $\sigma(5, k)$. For instance, $\delta(5,6)=1, \gamma(5,6)=5$ of which two are stars so $\sigma(5,6)$ $=2$. In terms of these numbers one can then express the cluster integrals and the virial coefficients as follows:

$$
\begin{aligned}
& b_{l}=\frac{1}{l ! V} \sum_{k=l-1}^{l l(l-1)} \sum_{i=1}^{\gamma(l, k)} c_{\imath}(l, k) \Im_{c}(\imath)(l, k), \\
& B_{l}=-\frac{(l-1)}{l ! V} \sum_{k=l}^{\frac{1}{2} l(l-1)} \sum_{i=1}^{\sigma(l, k)} s_{2}(l, k) \oiint_{s}^{(\imath)}(l, k) .
\end{aligned}
$$

$\Im_{c^{(\imath)}}(l, k)$ and $\Im_{s}{ }^{(\imath)}(l, k)$ are the irreducible integrals for the connected graph and star of $l$ points, $k$ lines, and of the $i$ 'th topological type.

\section{THE NUMBERS $d(l, k), c(l, k)$, AND $s(l, k)$}

Let us begin with the separated configurations. Since a separated configuration is made up of a number (at least two) of connected graphs, and since the numbers $d(l, k)$ and $c(l, k)$ are related by Eq. (11), it is evident that one must be able to express $d(l, k)$ in the $d$ 's involving smaller numbers of particles. To formulate this relation, let the separated configuration of $l$ points and $k$ lines consist of $n_{1}$ isolated points, $n_{2}$ connected pairs of points, $n_{3}$ connected triples of points, etc. Let $j$ be an index which distinguishes between the $n_{\imath}$ different connected graphs of $i$ particles, and let $k_{\imath \jmath}$ be the number of lines in the $j$ 'th graph. Clearly one must have:

$$
\sum_{\imath=1}^{l-1} i n_{\imath}=l, \quad \sum_{\imath=2}^{l-1} \sum_{j=1}^{n_{\imath}} k_{\imath \jmath}=k
$$

The upper limit of $i$ is $l-1$, since one must have at least one point separated from the rest of the graph. One then

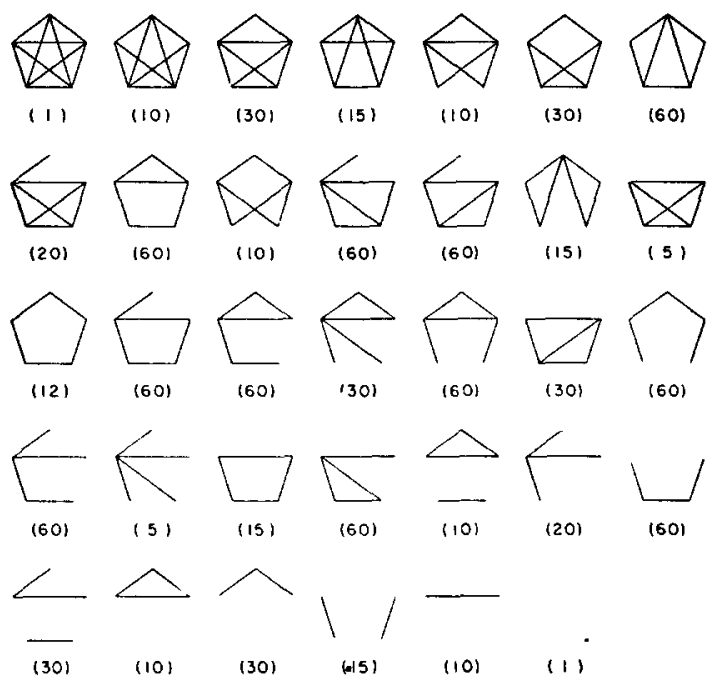

FIG. 1. Topologically different graphs of five points.

can convince oneself that:

$$
\begin{aligned}
d(l, k)=S_{\left(n_{\imath}\right)}^{\prime} \prod_{\imath=1}^{l-1} & \frac{l !}{(i !)^{n_{2}} n_{\imath} !} \\
& \times \underset{\left(k_{i \jmath}\right)}{S^{\prime}} \prod_{j=1}^{n_{i}}\left\{\left(\begin{array}{c}
\frac{1}{2} i(i-1) \\
k_{\imath \jmath}
\end{array}\right)-d\left(i, k_{\imath}\right)\right\}
\end{aligned}
$$

where the round summation signs go over all sets of numbers $n_{\imath}$ and $k_{i j}$ consistent with the relations (14), of which one is reminded by the primes. One can remove these restrictions by multiplying (15) by $x^{l} y^{k}$, and one can then write:

$$
\begin{aligned}
& \frac{d(l, k)}{l !}=\text { coefficient of } x^{l} y^{k} \text { in: } \\
& \underset{\left(n_{\imath}\right)}{S} \prod_{\imath=1}^{l-1} \frac{1}{(i !)^{n_{\imath}} n_{\imath} !} S_{\left(k_{\imath \jmath}\right)} \prod_{j=1}^{n_{i}}\left\{\left(\begin{array}{c}
\frac{1}{2} i(i-1) \\
k_{\imath \jmath}
\end{array}\right)-d\left(i, k_{\imath \jmath}\right)\right\} x^{2} y^{k_{\imath}} .
\end{aligned}
$$

The sums over the $k_{\imath \jmath}$ are readily carried out, to give:

$$
\begin{aligned}
& \frac{d(l, k)}{l !}=\text { coefficient of } x^{l} y^{k} \text { in: } \\
& S_{\left(n_{i}\right)} \prod_{l=1}^{\imath=1} \frac{1}{n_{\imath} !}\left\{(1+y)^{\frac{1}{2}(\imath-1)} \frac{x^{2}}{i !}-\sum_{k=0}^{\frac{1}{(i-1)(\imath-2)}} \frac{d(i, k)}{i !} x^{i} y^{k}\right\}^{n_{\imath}} .
\end{aligned}
$$

If one adds and subtracts the term for $i=l$, then the product may be taken to infinity since terms with $i>l$ will not contribute to the coefficient of $x^{l}$. One thus obtains :

$$
\begin{aligned}
& \frac{1}{l !}\left(\begin{array}{c}
\frac{1}{2} l(l-1) \\
k
\end{array}\right)=\text { coefficient of } x^{l} y^{k} \text { in: } \\
& \quad \exp \left[\sum_{\imath=1}^{\infty} \frac{x^{\imath}}{i !}(1+y)^{\frac{1}{\imath \imath(\imath-1)}-}-\sum_{\imath, k} \frac{d(i, k)}{i !} x^{i} y^{k}\right]
\end{aligned}
$$


Introducing therefore the generating functions:

$$
N(x, y)=\sum_{i=1}^{\infty} \frac{x^{2}}{i !}(1+y)^{\frac{1}{2(2-1)}}
$$

and

$$
D(x, y)=\sum_{l=1}^{\infty} \sum_{k} \frac{d(l, k)}{l !} x^{l} y^{k}
$$

where the summation over $k$ goes from zero to $\frac{1}{2}(l-1)(l-2)$, the greatest number of lines which can occur in a separated configuration, one obtains the simple relation: $:^{4}$

$$
D(x, y)=N(x, y)-\ln \{1+N(x, y)\} .
$$

From this one easily derives the explicit expression:

$$
d(l, k)=\sum_{r=2}^{l} \frac{(-1)^{r}}{r} S_{\left(l_{2}\right)}^{\prime} \frac{l !}{l_{1} ! l_{2} ! \cdots l_{r} !}\left(\begin{array}{c}
\sum_{i=1}^{r} \frac{1}{2} l_{\imath}\left(l_{2}-1\right) \\
k
\end{array}\right),
$$

where in the round sum the $l_{\imath}$ all start from one and the prime means the condition $l_{1}+l_{2}+\cdots+l_{r}=l$. For $k$ near the upper limit, $\frac{1}{2}(l-1)(l-2)$, only the terms with $r=2, l_{1}=1, l_{2}=l-1$, or vice versa, contribute, so that for $k>\frac{1}{2}(l-2)(l-3)+1$ :

$$
d(l, k)=l\left(\begin{array}{c}
\frac{1}{2}(l-1)(l-2) \\
k
\end{array}\right) .
$$

For smaller $k$ one gets corrections to this number, the first of which are:

$$
\begin{aligned}
\frac{1}{2} l(l-1) & \left(\begin{array}{c}
\frac{1}{2}(l-2)(l-3)+1 \\
k
\end{array}\right) \\
-l(l-1) & \left(\begin{array}{c}
\frac{1}{2}(l-2)(l-3) \\
k
\end{array}\right) .
\end{aligned}
$$

For large $l$ this can be written:

$$
d(l, k) \cong l\left(\begin{array}{c}
\frac{1}{2}(l-1)(l-2) \\
k
\end{array}\right)\left[\begin{array}{c}
l \\
1-\frac{-e^{-2 k / l}}{2}+\cdots
\end{array}\right],
$$

so that for $k>l \log l$ the correction goes to zero for $l \rightarrow \infty$. In the main range of values for $k$, Eq. (18) gives the asymptotic behavior for large $l$ of the number of separated configurations. Only for small $k$, for which $d(l, k)$ is small anyway, does Eq. (18) fail for large $l$.

Turning now to the numbers of stars, $s(l, k)$, among the connected graphs of $l$ points and $k$ lines, we first derive a recurrence relation for the number of connected graphs, $c(l, k)$, which is based on the idea that any connected graph can be considered to be built up out of stars which are connected to each other at the articulation points. To formulate this more precisely, let the

\footnotetext{
${ }^{14}$ We assume the convention that $d(1,0)=0$.
}

connected graph consist of $n_{2}$ stars of two points (bars), $n_{3}$ stars of three points (triangles), $n_{4}$ stars of four points (rectangles with zero, one or two diagonal lines), etc. Let $j$ distinguish between the $n_{2}$ different stars of $i$ points, and let $k_{23}$ be the number of lines in the $j^{\prime}$ th star. One must have of course :

$$
\sum_{\imath=2}^{l}(i-1) n_{\imath}=l-1, \quad \sum_{\imath=2}^{l} \sum_{\imath=1}^{n \imath} k_{\imath \jmath}=k
$$

The upper limit for $i$ is $l$ since the connected graph may just be one star of points. Similarly to Eq. (15), one now has:

$$
c(l, k)=\underset{\left(n_{2}\right)}{S^{\prime}} H\left(n_{2}, n_{3}, \cdots\right) \underset{\left(k_{2}\right)}{S^{\prime}} \prod_{l=1}^{l} \prod_{j=2}^{n 2} s\left(i, k_{\imath_{3}}\right),
$$

where $H\left(n_{2}, n_{3}, \cdots\right)$ is the number of Husimi trees of type $n_{2}, n_{3}, \cdots$ given by Eq. (10), and the two primes refer to the two conditions (21). Removing these conditions again by multiplying with $x^{l-1} y^{k}$, one obtains:

$c(l, k)=$ coefficient of $x^{l-1} y^{k}$ in:

$$
\frac{l !}{l^{2}} \exp \left\{l \sum_{i=2}^{\infty} \sum_{k=\imath}^{\frac{1}{2} \imath(\imath-1)} \frac{s(i, k)}{(i-1) !} x^{\imath-1} y^{k}\right\} .
$$

If we now introduce the generating function:

$$
S(x, y)=\sum_{l=2}^{\infty} \sum_{k=l}^{\frac{1}{2} l(l-1)} \frac{s(l, k)}{l !} x^{l} y^{k},
$$

and use Eq. (11), one can write (23) in the form:

$$
\begin{gathered}
\left(\begin{array}{c}
\frac{1}{2} l(l-1) \\
k
\end{array}\right)-d(l, k)=\text { coefficient of } x^{l-1} y^{k} \text { in: } \\
\frac{l !}{l^{2}} \exp \left\{\frac{\partial S(x, y)}{\partial x}\right\} .
\end{gathered}
$$

If we multiply this equation by $(l / l !) x^{l} y^{k}$, sum over all $l$ and $k$, and use Eq. (16), we get:

$$
z(x, y)=\sum_{l=1}^{\infty} \frac{x^{l}}{l !}\left\{\frac{d^{l-1}}{d \xi^{l-1}} \exp \left[\frac{\partial S(\xi, y)}{\partial \xi}\right]\right\}_{\xi=0},
$$

where $z(x, y)$ is defined as:

$$
z(x, y)=x \frac{\partial}{\partial x} \ln \{1+N(x, y)\} .
$$

From Lagrange's theorem ${ }^{15}$ then follows:

$$
\frac{\partial S(z, y)}{\partial z}=\stackrel{z}{z}-
$$

${ }^{15}$ See for instance Whittaker and Watson, Modern Analysis, Chap VII (Macmillan, New York, 1944). In order that Eq. (26) is valid for all $l$ and $k$, we assume the following conventions: $s(1,0)=0, c(1,0)=1 ; s(2,1)=c(2,1)=1$. 
It is not possible to obtain from (25) and (26) an explicit expression for $s(l, k)$, since expressing $x$ in terms of $y$ and $z$ through (25) involves the inversion of a power series. However, one can obtain an asymptotic expression for large $l$. To do this it is easier to start from Eq. (24). By developing the exponential function and by taking in the different powers of $l \partial S / \partial x$ always the coefficients of the highest powers of $y$, one obtains a recurrence relation of the form:

$$
\begin{aligned}
&\left(\begin{array}{c}
\frac{1}{2} l(l-1) \\
k
\end{array}\right)- d(l, k)=s(l, k)+l(l-1) s(l-1, k-1) \\
&+\frac{1}{2} l(l-1)(l-2) s(l-2, k-3) \\
& \quad+\frac{1}{2} l(l-1)(l-2) s(l-2, k-2)+\cdots,
\end{aligned}
$$

in which the further terms depend on the number of stars with less than $l-2$ points. From this one sees that in first approximation for large $l$ :

$$
s(l, k) \cong\left(\begin{array}{c}
\frac{1}{2} l(l-1) \\
k
\end{array}\right) .
$$

In second approximation, using (18):

$$
\begin{aligned}
s(l, k) \cong\left(\begin{array}{c}
\frac{1}{2} l(l-1) \\
k
\end{array}\right)-l\left(\begin{array}{c}
\frac{1}{2}(l-1)(l-2) \\
k
\end{array}\right) & \\
& -l(l-1)\left(\begin{array}{c}
\frac{1}{2}(l-1)(l-2) \\
k-1
\end{array}\right) .
\end{aligned}
$$

This expression is exact for $k>\frac{1}{2}(l-2)(l-3)+1$. In the next approximation one finds further correction terms which contribute for smaller values of $k$. Just as for the $d(l, k)$, we can conclude that for large $l$ and almost all $k$ (in fact again for $k>l \log l$ ), Eq. (27) will give the number of stars, or in other words for large $l$ practically all graphs of $k$ lines are stars. From Eq. (28) one can see further that for large $l$ the number of connected graphs with at least one articulation point is asymptotically:

$$
l(l-1)\left(\begin{array}{c}
\frac{1}{2}(l-1)(l-2) \\
k-1
\end{array}\right) .
$$

\section{THE NUMBERS $\delta(l, k), \gamma(l, k)$, and $\sigma(l, k)$.}

The first problem is to determine the total number:

$$
\pi(l, k)=\delta(l, k)+\gamma(l, k),
$$

of topologically different graphs of $l$ points and $k$ lines, which is the analogue of the binomial coefficient $\left(\frac{1}{2} l(l-1), k\right)$ in Eq. (11). This problem has been solved by Pólya. ${ }^{16} \mathrm{He}$ expresses his result in terms of the

\footnotetext{
${ }^{16}$ We are greatly indebted to Professor G. P6lya for communicating his result to us. In this section we will omit the proofs, since they would take up too much space, and since we hope that they will appear elsewhere. They can be found in the dissertation of R. J. Riddell (Ann Arbor, 1951, p. 57.)
}

generating function:

$$
F_{l}(y)=\sum_{k} \pi(l, k) y^{k}
$$

and he finds:

$$
\begin{aligned}
& F_{l}(y)=\underset{\left({ }^{\prime}\right)}{S^{\prime}} \frac{1}{\prod_{r} j_{r} ! r^{\jmath r}} \prod_{r=\text { odd }}\left(1+y^{r}\right)^{\frac{1}{2}(r-1)_{2 r}} \\
& \text { - } \prod_{r=\mathrm{even}}\left[\left(1+y^{r / 2}\right)\left(1+y^{r}\right)^{\frac{1}{2}(r-2)}\right]^{\prime r} \\
& \cdot \prod_{r}\left(1+y^{r}\right)^{\frac{1}{r} j_{r}\left(\jmath_{r}-1\right)} \cdot \prod_{r<s}\left(1+y^{m(r, s)}\right)^{t(r, s) \jmath_{r} \geq s} .
\end{aligned}
$$

Here the round summation sign goes over all sets of integers $j_{r}$, starting from zero, which fulfill the condition:

$$
\sum_{r=1}^{l} r j_{r}=l \text {. }
$$

$m(r, s)$ is the least common multiple and $t(r, s)$ the greatest common divisor of $r$ and $s$. Take for example $l=5$; the sum consists then of seven terms corresponding to the seven partitions $5=1+1+1+1+1,5=1+1+1$ $+2,5=1+1+3,5=1+2+2,5=1+4,5=2+3,5=5$, for which one finds:

$$
\begin{gathered}
5 ! F_{5}(y)=(1+y)^{10}+(1+y)^{4}\left(1+y^{2}\right)^{3}+20(1+y)\left(1+y^{3}\right)^{3} \\
+15(1+y)^{2}\left(1+y^{2}\right)^{4}+30\left(1+y^{2}\right)\left(1+y^{4}\right)^{2} \\
+20(1+y)\left(1+y^{3}\right)\left(1+y^{6}\right)+24\left(1+y^{5}\right)^{2} .
\end{gathered}
$$

This leads to:

$$
\begin{aligned}
F_{5}(y)=1+y+2 y^{2}+4 y^{3}+6 y^{4} & +6 y^{5} \\
& +6 y^{6}+4 y^{7}+2 y^{8}+y^{9}+y^{10},
\end{aligned}
$$

from which one can read off $\pi(5, k)$, which can be checked with Fig. 1.

From Eq. (31) one can also derive the asymptotic behavior of $\pi(l, k)$ for large $l$. Taking only the partitions of $l$ in $l$ one's and in $l-2$ one's and one two, one gets:

$$
\begin{aligned}
& l ! F_{l}(y)=(1+y)^{\frac{1}{2} l(l-1)} \\
& +\frac{1}{2} l(l-1)(1+y)^{\frac{l}{(l-2)(l-3)+1}\left(1+y^{2}\right)^{l-2}+\cdots}
\end{aligned}
$$

from which one sees that for large $l$ the main contribution to $\pi(l, k)$ comes from the first term, so that:

$$
\pi(l, k) \cong \frac{1}{l !}\left(\begin{array}{c}
\frac{1}{2} l(l-1) \\
k
\end{array}\right) .
$$

From the second term in (32) one can find corrections to this result, which however vanish for large $l$ and for $k$ not too near the two ends of its range, zero and $\frac{1}{2} l(l-1) .^{17}$

\footnotetext{
${ }^{17}$ We are indebted to Dr. P. Erdös for communicating to us various estimates for the range of $k$ for which our asymptotic results are valid. With regard to Eq. (33) he thinks that $k$ must lie in the range
}

just as for Eqs. (18) and (27). 
The next question is the determination of the number $\delta(l, k)$ of separated configurations, which are topologically different. The answer ${ }^{18}$ can be expressed as follows: Define the double generation functions as

$$
\begin{aligned}
& P(x, y)=\sum_{l=0}^{\infty} F_{l}(y) x^{l}=\sum_{l} \sum_{k} \pi(l, k) x^{l} y^{k}, \\
& \Delta(x, y)=\sum_{l} \sum_{k} \delta(l, k) x^{l} y^{k} .
\end{aligned}
$$

Then one can prove the relation

$$
\sum_{\nu} \underset{\nu}{1} \Delta\left(x^{\nu}, y^{\nu}\right)=\sum_{\nu} \underset{\nu}{1}\left\{P\left(x^{\nu}, y^{\nu}\right)+(-1)^{\nu} P^{\nu}(x, y)\right\}
$$

from which one can find the $\delta(l, k)$ in terms of the known $\pi(l, k)$. Especially one can show from Eq. (35) that for large $l$ and a suitable range of $k$, one has

$$
\delta(l, k) \cong \frac{1}{(l-1) !}\left(\begin{array}{c}
\frac{1}{2}(l-1)(l-2) \\
k
\end{array}\right) .
$$

We have not succeeded in splitting the number of topologically different stars $\sigma(l, k)$ from the number of connected graphs $\gamma(l, k)=\pi(l, k)-\delta(l, k)$. However, there is no doubt that for large $l, \sigma(l, k) \cong \pi(l, k)$, and that the number of topologically different connected graphs with at least one articulation point is asymptotically

$$
\frac{1}{l !} l(l-1)\left(\begin{array}{c}
\frac{1}{2}(l-1)(l-2) \\
k-1
\end{array}\right) .
$$

The reason is simply that for large $l$ the great majority of all graphs are stars as we saw in Sec. IV, and that only very rarely will a graph have some kind of symmetry. As a result for large $l$ the numbers $d_{\imath}(l, k), c_{\imath}(l, k), s_{\imath}(l, k)$ will be just $l$ ! for practically all $i$, so that (see Eq. (12)) asymptotically $\delta(l, k), \gamma(l, k)$, and $\sigma(l, k)$ will be $(1 / l !)$ times $d(l, k), c(l, k)$, and $s(l, k)$.

Finally we will consider a few special cases, which have some interest for themselves and which will illustrate further the relation between the numbers $s(l, k), s_{\imath}(l, k)$, and $\sigma(l, k)$. First consider the case $k=l$. All stars are in the form of a ring, so $\sigma(l, l)=1$. The points can be put on the ring in any order, which gives a factor $l$ ! in $s(l, l)$. However we may choose any point as the starting point, and we may count either clockwise or counter-clockwise around the ring without changing the configuration. This gives a "symmetry number" $2 l$

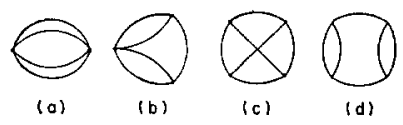

Fig. 2. Homeomorphic types of graphs for $k=l+2$.

${ }^{18}$ For the proof, see R. J. Riddell, dissertation, University of Michigan, 1951, p. 65. by which the $l$ ! has to be divided. So:

$$
s(l, l)=\frac{1}{2}(l-1) \text { ! }
$$

If $k=l+1$ the stars take the form of three chains connected at their ends, in which there may be $l_{1}$ particles in the first chain, $l_{2}$ in the second, and $l_{3}$ in the third (not counting the end particles), so that $l_{1}+l_{2}+l_{3}=l-2$. Not more than one chain may have $l_{2}=0$. Any partition therefore of $l-2$ in two or three summands will correspond to a star of a definite topological type. The number $\sigma(l, l+1)$ is the number of such partitions and these can be found by standard methods. One can show that $\sigma(l, l+1)$ is the coefficient of $y^{l-2}$ in

$$
\frac{1}{(1-y)\left(1-y^{2}\right)\left(1-y^{3}\right)}-\frac{1}{1-y},
$$

and explicit expressions can easily be obtained. Especially for large $l$ one finds that $\sigma(l, l+1) \cong l^{2} / 12$. To find $s_{\imath}(l, l+1)$, note that if all chains are different there is only a mirror symmetry between left and right, but if some chains are alike, then these chains may be interchanged as well. Thus we obtain:

$$
s_{\imath}(l, l+1)=l ! / 2 n_{\imath} !
$$

where $n_{3}$ is the number of chains which are alike. Since for large $l$ it will be a rare case if two or more chains are alike, it is clear that asymptotically $s(l, l+1) \cong l ! l^{2} / 24$, and it is also not difficult to show that exactly

$$
s(l, l+1)=(l ! / 24)(l-3)(l-2) .
$$

The fact that for $k=l+1$ all stars take the form of three chains is expressed in the theory of graphs by saying that all stars are homeomorphic to each other. If $k$ increases, the number of homeomorphic types increases too. For instance for $k=l+2$ there are four homeomorphisms, as shown in Fig. 2. To find for each type the number of topologically different stars, is again a partition problem of $l-2, l-3$, or $l-4$ in four, five, or six parts. The $s_{2}(l, l+2)$ are $l ! / s_{\imath}$ where $s_{\imath}$ is the number of symmetry operations which may be performed on the star without changing it. For instance if in the four types (a), (b), (c), and (d) the chains are all alike $s_{\imath}$ will have the values $2 \cdot 4 !(a) ; 2^{3}(b) ; 4 !(c) ; 2^{4}(d)$. Finally by summing over all partitions one can determine $s(l, l+2)$ for each of the four types. We will not write down the explicit numbers, and mention only that altogether

$$
s(l, l+2)=\frac{l !}{48 \cdot 4 !}(l-3)\left(l^{4}+4 l^{3}-15 l^{2}-46 l-40\right) .
$$

\section{REMARKS ON THE INTEGRAL PROBLEM}

1. If the intermolecular force is always repulsive the $f_{23}(r)$ of Eq. (2) will always by negative. Hence the sign of all the irreducible integrals $\Im^{(\imath)}(l, k)$ will be $(-1)^{k}$. With regard to the absolute magnitude it is clear that the star integrals must decrease with increasing $k$, be- 
cause adding a line between two points means that we introduce a factor in the integrand which requires the two points to be less than a certain distance apart. Thus with increasing $k$ the integrand will differ from zero over a smaller region of the $3 l$ dimensional phase space. This is illustrated for the three stars of four points by the following known results for the integrals: $:^{19}$

$$
\begin{aligned}
& \square=2.5905 b^{3} ; \frac{16}{3} b^{3}, \\
& \square=-1.8894 b^{3} ;-\frac{14}{3} b^{3}, \\
& \square=1.2728 b^{3} ; \frac{12}{3} b^{3} .
\end{aligned}
$$

The second column gives the values of the integrals in one dimension; $b$ is the van der Walls' $b$ equal to four times the proper volume of a molecule in three dimensions, and equal to just the volume in one dimension.

2. In three dimensions the cluster integrals $b_{l}$ and virial coefficients $B_{l}$ for a gas of elastic spheres are only known up to $l=4$. In one dimension on the other hand one knows that:

$$
\begin{aligned}
& B_{l}=b^{l-1}, \\
& b_{l}=\frac{(-1)^{l-1}}{l !} b^{l} .
\end{aligned}
$$

Note that the $B_{l}$ are all positive and the $b_{l}$ are alternating in sign. Whether this is also so in three dimensions is not known, but is plausible.

One might hope to gain insight into the relation between the numbers $s(l, k)$ and the integral $\mho_{s}(l, k)$ by considering the one-dimensional case in greater detail. For all configurations the integrals are elementary and can be calculated successively. For instance for $l=5$ the results for $\Im_{8}^{(\imath)}(l, k)$ together with the $s_{\imath}(l, k)$ are given in Table I. It would be of interest to do the calculation for arbitrary $l$ and $k$, and to see especially what the asymptotic behaviour of the $\oiint_{s}(l, k)$ is for large $l$. But we have not succeeded in doing that. ${ }^{20}$

3. Because of the alternating sign of the $\oiint_{s}(l, k)$ for repulsive forces the alternating moments of the $s(l, k)$ may be of interest. These can all be calculated from the general formulas, Eq. (25) and Eq. (26). Defining

$$
M_{s}^{(n)}(l)=\sum_{k=l}^{\frac{1}{3} l(l-1)}(-1)^{k} k^{n} S(l, k),
$$

\footnotetext{
${ }^{19}$ The irreducible integrals in two dimensions have also been evaluated by Harris, Selis. and Guth (to be published).

\begin{tabular}{|c|c|c|c|c|c|c|c|c|c|c|}
\hline TYPE & 0 & $\Delta$ & $\otimes$ & $\triangle$ & $\otimes$ & $\otimes$ & $\mathbb{A}$ & $\Theta$ & $\otimes$ & $\notin$ \\
\hline$t^{-4} \times 8_{s}^{(1)}(5, k$ & $-\frac{115}{12}$ & $+\frac{49}{6}$ & +8 & $-\frac{29}{4}$ & $-\frac{41}{6}$ & $-\frac{15}{2}$ & +6 & $+\frac{19}{3}$ & $-\frac{11}{2}$ & \\
\hline$S_{1}(5, k)$ & 12 & 60 & 10 & 60 & 30 & 10 & 15 & 30 & 10 & \\
\hline
\end{tabular}

${ }^{20}$ Using Eq. (13) one finds from Table I $B_{5}=b^{4}$ in agreement with (42) For $l=4$ the numbers $s(l, k)$ are $3,6,1$ and from (41) then follows $B_{4}=b^{3}$, again in agreement with (42).
}

TABLE I. Decomposition of the 5 th virial coefficient for onedimensional rigid lines.

one finds for instance:

$$
\begin{aligned}
& M_{8}{ }^{(0)}(l)=-(l-2) ! \\
& M_{8}{ }^{(1)}(l)=-\frac{1}{2} l !, \\
& M_{s}^{\left({ }^{2}\right)}(l)=-\frac{1}{8} l !\left(l^{2}+3 l-6\right) .
\end{aligned}
$$

Analogous formulas can be found for the alternating moments of $d(l, k)$ and therefore of $c(l, k)$. However, we have not been able to exploit these results for the general problem.

4. Since for repulsive forces the $f_{\imath}$, have always the same sign one may interpret $f_{2,}$ as the (unnormalized) probability of going from the point $i$ to the point $j$. For a linear chain of $n$ particles the integral,

$$
I\left(\mathbf{r}_{1}, \mathbf{r}_{n}\right)=\int \cdots \int d^{3} r_{2} \cdots d^{3} r_{n-1} f_{12} f_{23} \cdots f_{n-1, n},
$$

can then be considered as the probability of going from $\mathbf{r}_{1}$ to $\mathbf{r}_{n}$ by a random walk of $n-1$ steps, and from the central limit theorem one knows that $I\left(\mathbf{r}_{1}, \mathbf{r}_{n}\right)$ will become a Gaussian distribution for large $n$. In fact for the case of elastic spheres $\left(f\left(\left|\mathbf{r}_{\imath}-\mathbf{r}_{\jmath}\right|\right)=-1\right.$ for $\left|\mathbf{r}_{\imath}-\mathbf{r}_{\jmath}\right|<d$ and zero otherwise) one easily shows that asymptotically

$$
I\left(\mathbf{r}_{1}, \mathbf{r}_{n}\right) \cong(-1)^{n} \frac{(2 b)^{n}}{(2 \pi)^{3}}\left(\frac{10 \pi}{n d^{2}}\right)^{\frac{3}{2}} \exp \left(-\frac{5 r^{2}}{2 n d^{2}}\right),
$$

with $b=2 \pi d^{3} / 3$ and $r=\left|\mathbf{r}_{1}-\mathbf{r}_{n}\right|$. For $n=3$, Eq. (45) already gives a close approximation.

If, therefore, the star consists of a number of connected chains, which are long enough so that (45) can be applied, then the integrand of the star integral becomes a Gaussian distribution in the linkage points of the chains, and the evaluation becomes straightforward. ${ }^{21}$ For instance, for the ring integrals $\oiint_{s}(l, l)$ one gets simply:

$$
\Im_{s}(l, l) \cong I(0)=(-1)^{l} \frac{(2 b)^{l-1}}{l^{l}} \frac{5}{3}\left(\frac{10}{\pi}\right)^{\frac{1}{2}},
$$

so that, if one writes Eq. (13) in the form

$$
B_{l}=\sum_{k=l}^{\frac{1}{2} l(l-1)} B(l, k)
$$

${ }^{21}$ The fact that for a single chain the integral (44) can always be reduced to a single integral by a Fourier transformation (since (44) is obtained by folding the function $f(r)(n-1)$ times) was first noted by E W. Montroll and J. E. Mayer [J. Chem. Phys 9, 626 (1941) ]. Also Eq. (45) and the results for the ring and three chain integrals can be found essentially in this paper. 
one gets, using (38):

$$
B(l, l) \cong(-1)^{l-1} \frac{l-1}{l^{\frac{5}{4}}}(2 b)^{l-1} \frac{5}{3}\left(\frac{5}{2 \pi}\right)^{\frac{1}{2}}
$$

For $l=3$, this differs from the well known exact value $5 b^{2} / 8$ by 22 percent; for $l=5$, the error is only 12 percent. For the case $k=l+1$, where the stars take the form of three chains linked at their ends, the integral becomes

$$
\begin{aligned}
& \oiint_{s}^{(\imath)}(l, l+1) \cong(-1)^{l+1} \frac{250}{9 \pi}(2 b)^{l-1} \\
& \quad \times\left[\left(l_{1}+1\right)\left(l_{2}+1\right)+\left(l_{2}+1\right)\left(l_{3}+1\right)+\left(l_{3}+1\right)\left(l_{1}+1\right)\right]^{-\frac{2}{2}},
\end{aligned}
$$

where $l_{1}, l_{2}, l_{3}$ are the numbers of particles in the chains, so that $l_{1}+l_{2}+l_{3}=l-2$. Using the result of Sec. V on the $s_{\imath}(l, l+1)$, one can show that for large $l$ :

$$
B(l, l+1)=(-1)^{l} \frac{250}{9}(2 b)^{l-1} .
$$

In principle one can go on in this way. For $k=l+2$ one has to consider the four homeomorphisms shown in Fig. 2, and the calculation (especially the summation over all the numbers of particles in the different chains) becomes rapidly more involved, so that we have not been able to go further.

\title{
Dynamics of the Graphite Lattice*
}

\author{
HERBERT B. Rosenstock \\ U. S. Naval Research Laboratory, Washington, D. C.
}

(Received July 7, 1953)

\begin{abstract}
The distribution of frequencies of normal vibrations in the graphite lattice has been obtained in closed form for low temperatures in the two-dimensional approximation for the values $(\alpha, \beta, 0)$ and $(\alpha, \beta, \alpha / 2)$ of the force constants $(\alpha, \beta, \gamma)$ for nearest, second-nearest, and third-nearest neighbors. The frequencies run from 0 to a finite value $\omega_{\max }$, with two logarithmic peaks in their density. The specific heat has been calculated for two special sets of force constant ratios. Deviations from $T^{2}$ dependence set in at temperatures much lower than predicted by Debye theory. Comparison with experiment leads to a numerical value for $\theta=\hbar \omega_{\max } / k$, from which $\omega_{\max }$, and therefore also $\alpha$ and one of the macroscopic elastic constants, $c_{44}$, may be calculated. $\theta$ and $\omega_{\max }$ appear very sensitive to the assumed force constant ratios, $c_{44}$ less so.
\end{abstract}

\section{INTRODUCTION}

$\mathrm{O}$ NE plane in a graphite lattice is shown in Fig. 1. On account of the wide separation of planes, it is believed $^{1}$ that the interaction between atoms in different planes is so slight that each crystallite consists of only a few (12-17) planes. It follows that no vibrational waves will be propagated perpendicular to the planes (or, at any rate, none with a wavelength long enough to be of importance at low temperatures). Gurney ${ }^{1}$ further argues that of the remaining possible waves only those that are propagated in the planes but consist of vibrations perpendicular to the planes will be appreciably excited at low temperatures, since the restoring forces for "in-plane" motion will be much greater than for "transverse" motion. The principal object of Gurney's paper is to point out that the two-dimensional problem so resulting leads to a $T^{2}$-law (instead of the $T^{3}$-law obtained for three-dimensional solids) for the specific heat at low temperatures if the Debye approximation is used for the frequency distribution, and that this agrees with experiment.

It is, however, possible to calculate the frequency distribution of transverse vibrations of the two-dimen-

\footnotetext{
* Results reported at the North Carolina meeting of the American Physical Society, March, 1953 (H. B. Rosenstock, Phys. Rev. 91, 233(A) (1953).

${ }^{1}$ R. W. Gurney, Phys. Rev. 88, 465 (1952).
}

sional "graphite" lattice exactly for certain values of the force constants $\alpha, \beta$, and $\gamma$ for nearest, 2nd-nearest, and 3rd-nearest neighbors. The purpose of this paper is, then, to carry out this calculation where possible, to calculate the specific heat using several special cases of this exact distribution, rather than using the approximate Debye distribution; and by comparison with experiment to try to infer the values of the force constants and the related "elastic constants" which should in principle be experimentally measurable.

\section{THE FREQUENCIES}

Let $w_{\imath}$, be the displacement, perpendicular to the plane, of atom $i j$ of Fig. 1, and $m$ be the mass of each atom. The kinetic energy of the lattice of $N^{2}$ particles is then

$$
T=\frac{1}{2} m \sum_{\text {all } 2, j}^{N, N} \dot{w}_{\imath j}^{2}
$$

and the potential energy

$$
\begin{aligned}
& V=\frac{1}{2} \alpha \sum_{\substack{\text { nearest } \\
\text { neighbors }}}\left(w_{2 j}-w_{k l}\right)^{2} \\
& \quad+\frac{1}{2} \beta \sum_{\substack{\text { 2nd } \\
\text { nearest }}}\left(w_{2}-w_{k l}\right)^{2}+\frac{1}{2} \gamma \sum_{\substack{\text { 3rd } \\
\text { nearest }}}\left(w_{2 j}-w_{k l}\right)^{2}
\end{aligned}
$$

